\title{
Autoradiographic Investigation of Cell Proliferation in the Brain of Spontaneously Hypertensive Rats
}

\author{
Fumitada Hazama, Hideyuki Haebara, Shigeru \\ Amano, and Takashi Ozaki*
}

Cell proliferation in the brain of spontaneously hypertensive rats and control Wistar rats of various ages was autoradiographically investigated using ${ }^{3} \mathrm{H}$-thymidine.

The brain of SHR showed a definite increase of labelled cells in number when compared with the control animals. The increase of labelled cells was observed already at 8 weeks after birth, in the early stage of hypertension. And the labelled cells showed a tendency to increase in number according to the age of animals.

The distribution of the labelled cells in the brain corresponds with those of increased vascular permeability demonstrated by horseradish peroxidase and cerebrovascular lesions.

The labelled cells consisted of endothelial cells of the intracerebral arterioles and capillaries, perivascular fibroblasts and glial cells in the brain parenchyma. Arachnoid cells, subarachnoidal fibroblasts, pial cells, endothelial, medial, and adventitial cells of the pial arteries were also labelled in small number. The labelling of endothelial cells of the intracerebral arterioles and capillaries indicates compensatory cell renewal for destruction of the cells caused by hypertension. The labelling of glial cells and perivascular fibroblasts in the early stage of hypertension seems to indicate a reactive proliferation of the cells to the increased vascular permeability, or edema, based on the endothelial changes.

(This research is supported in part by a Grant-in-aid for Scientific Research from Ministry of Education, and Science and Technology Agency, Japan.)

From the Departments of Pathology and Neurosurgery, ${ }^{*}$ Faculty of Medicine, Kyoto University, Kyoto. 